\title{
Lorentz Force Electrical Impedance Tomography
}

\author{
Pol Grasland-Mongrain ${ }^{1}$, Jean-Martial Mari ${ }^{1}$, Jean-Yves Chapelon ${ }^{1}$, Cyril Lafon ${ }^{1}$
}

1. Inserm, U1032, LabTau, Lyon, F-69003, France ; Université de Lyon, Lyon, F-69003, France

Résumé- This article describes a method called Lorentz Force Electrical Impedance Tomography. The electrical conductivity of biological tissues can be measured through their sonication in a magnetic field : the vibration of the tissues inside the field induces an electrical current by Lorentz force. This current, detected by electrodes placed around the sample, is proportional to the ultrasonic pressure, to the strength of the magnetic field and to the electrical conductivity gradient along the acoustic axis. By focusing at different places inside the sample, a map of the electrical conductivity gradient can be established. In this study experiments were conducted on a gelatin phantom and on a beef sample, successively placed in a $300 \mathrm{mT}$ magnetic field and sonicated with an ultrasonic transducer focused at $21 \mathrm{~cm}$ emitting $500 \mathrm{kHz}$ bursts. Although all interfaces are not visible, in this exploratory study a good correlation is observed between the electrical conductivity image and the ultrasonic image. This method offers an alternative to detecting pathologies invisible to standard ultrasonography.

\section{Mots-Clés}

Medical Imaging, Electrical Conductivity Imaging, Magneto-Acousto-Electrical Tomography, Ultrasonically-Induced Lorentz Force, Lorentz Force Electrical Impedance Tomography, Hall Effect Imaging

\section{INTRODUCTION}

The electrical conductivity of biological tissues arouses great interest for medical imaging researchers. Indeed this parameter potentially shows a good contrast in the human body. For example, fat is ten times less conductive than muscle tissue 5] while the acoustic impedance, observed in ultrasonography, only changes of a few percents through the soft tissues. Electrical conductivity anomalies can moreover reveal pathologies like tumors 8 .

The most advanced technique today to measure the electrical conductivity of tissue is the Electrical Impedance Tomography (EIT) [3. In this technique, several electrodes are placed around the body or the organ under study. An electrical current is injected through each electrode while its distribution in the tissues is measured by the others. These measurements allow an electrical impedance image reconstruction through mathematical analysis. This apparently simple and inexpensive technique suffers however of a low spatial resolution due to the intrinsic ill-posed nature of the mathematical problem [2].

On another hand, the vibration of a conductor inside a magnetic field induced by ultrasound induces by Lorentz force an electrical current which is connected to the electrical conductivity of the conductor 4. This approach can be applied to the measurement of ultrasound velocity using a wire as sensing element, for its electrical conductivity is known [6], 7]. Conversely,the ultrasound pressure distribution in the conductor is known [14, the electrical conductivity could be deduced . In this last approach, the biological tissues are submitted to a magnetic field created for example by a permanent magnet, and a focused ultrasound beam is used to vibrate the tissues in a specific region of interest 13 . In the same way the movement of a conductor in a magnetic field would induce an electrical current, this vibration induces a current in the tissues which can be detected by the mean of electrodes. The focusing of ultrasound allows conferring to the imaging process an important characteristic when compared to Electrical Impedance Tomography : the spatial resolution is close to the one that would be reached by ultrasound imaging. However, the drawback of such approach lies in the weakness of the induced electrical current. The initial name of the technique, the Hall Effect Imaging 21] was criticized later on as the phenomenon is not exactly an Hall Effect [18,. The technique has also been called Magneto-Acousto-Electrical Tomography [9] or scan of electric conductivity gradients with ultrasonically-induced Lorentz force [16]. We use here the name of Lorentz Force Electrical Impedance Tomography (LFEIT), which has the advantage of describing both the imaged parameter and the method.

\section{THEORY}

For clarity reasons, the $\mathrm{X}$ axis is defined as the orientation of the magnetic field, the $\mathrm{Z}$ axis is defined along the ultrasound propagation direction and the $\mathrm{Y}$ axis is placed accordingly using the right-hand rule. As expo- 


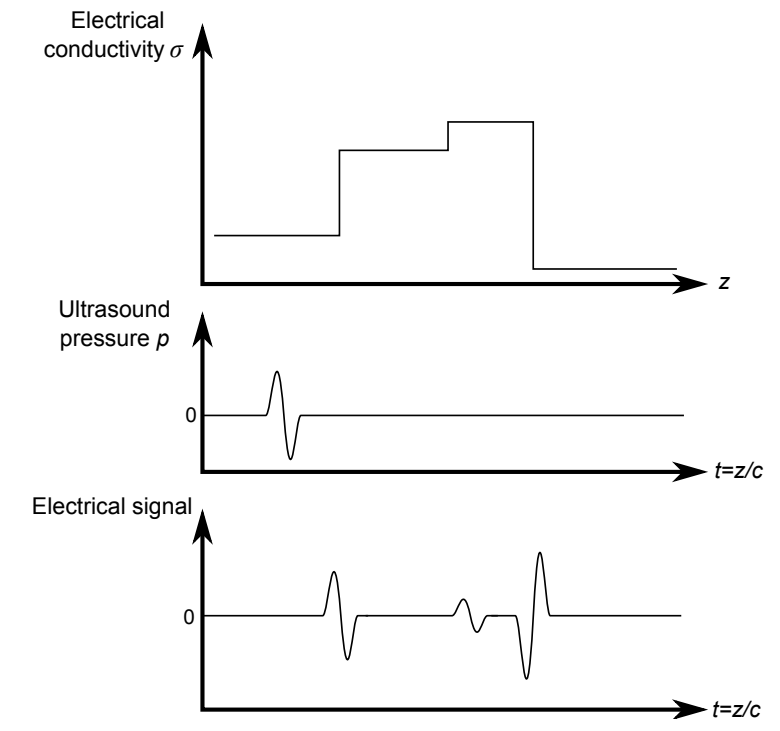

FiguRE 1 - The electrical signal detected by electrodes in Lorentz Force Electrical Impedance Tomography is proportional to the convolution product between electrical conductivity gradients and the ultrasound pressure shape.

sed previously, the Lorentz Force Electrical Impedance Tomography is based on the movement of a conductor placed inside a magnetic field, which induces an electrical current. Physical and mathematical modellings have been proposed to describe the method [1, 20]. We choose here the model presented by Montalibet et al. which gives a good understanding of the phenomenon [17. In this model, the induced current is found to be proportional to the convolution product of the electrical conductivity gradient $H$ with the ultrasound pressure shape $P$ :

$$
i(t)=\int\left(\frac{d \sigma}{d z} \frac{B}{\rho}\right) *\left(\int_{0}^{t} p(\tau) d \tau\right) d S=c(H * P)(t)
$$

with $i$ the induced electrical current, $\frac{d \sigma}{d z}$ the electrical conductivity gradient along $\mathrm{z}$ axis, $B$ the magnetic field, $\rho$ the density, $p$ the ultrasound pressure and $c$ the speed of sound.

In other words, the electrical current detected by the electrodes is a temporal image of the ultrasound pulse at each electrical conductivity interface, as shown by the figure (1). More refined models are however available [1, 20].

\section{MATERIALS AND METHODS}

The goal of this study was to build a setup to produces LFEIT images of gelatine phantoms and biological tissues.

The experiment setup is illustrated in figure (3). A generator (HP33120A, Agilent, Santa Clara, CA, USA)

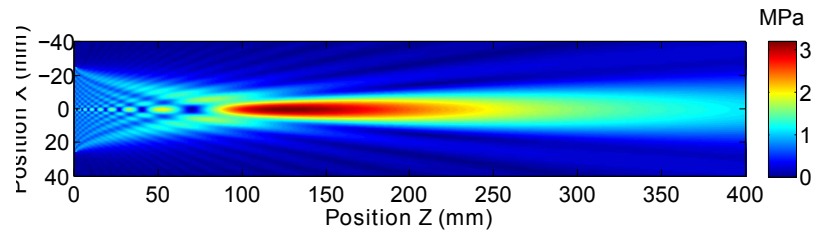

Figure 2 - Pressure field simulation along ultrasound axis $(Y=0 \mathrm{~mm})$ of the transducer. The maximum of pressure is approximately at $15 \mathrm{~cm}$ of the transducer.

created $0.5 \mathrm{MHz}, 3$ cycles sinusoid bursts at a pulse repetition frequency of $100 \mathrm{~Hz}$. This excitation was amplified by a $200 \mathrm{~W}$ linear power amplifier (200W LA200H, Kalmus Engineering, Rock Hill, SC, USA) and sent to a $0.5 \mathrm{MHz}, 50 \mathrm{~mm}$ in diameter transducer focused at $210 \mathrm{~mm}$ and placed in a $100 \times 50 \times 50 \mathrm{~cm}^{3}$ degassed water tank.

The ultrasound pressure was equal to $3 \mathrm{MPa}$ at the focal point $(\mathrm{Z}=210 \mathrm{~mm})$. A simulation of the ultrasound pressure field based on the resolution of the linear Rayleigh equation is shown in figure (2).

A $20 \times 4 \times 15 \mathrm{~cm}^{3}$ mineral oil tank was placed from 15 to $35 \mathrm{~cm}$ away from the transducer in the ultrasound beam axis. This oil tank was placed in the middle of a U-shaped permanent magnet, composed of two poles made of two $5 \times 5 \times 3 \mathrm{~cm} 3 \mathrm{NdFeB}$ magnets (BLS Magnet, Villers la Montagne, France). The gap between the poles was $4.5 \mathrm{~cm}$. The magnetic field was equal to 90 $\mathrm{mT}$ in a $4 \mathrm{~cm}$ radius circle around the maximum of $340 \mathrm{mT}$.

The tested sample was placed inside this tank from 22 to $28 \mathrm{~cm}$ of the transducer. Two samples were tested : a phantom made of $10 \%$ gelatin and $1 \%$ salt, $8 \mathrm{~cm}$ wide, as shown in figure (4); and a $6 \mathrm{~cm}$ wide piece of beef muscle with an L-shape, having a fat layer in the middle of the sample, as shown in figure (5). A pair of $10 \times 3 \times 0.1 \mathrm{~cm}^{3}$ copper electrodes was placed in contact with the sample, respectively above and under it. The electrodes were linked through an electrical wire to a $1 \mathrm{MV} / \mathrm{A}$ current amplifier (HCA-2M-1M, Laser Components, Olching, Germany) and an oscilloscope with $50 \Omega$ input impedance (WaveSurfer 422, LeCroy, Chestnut Ridge, NY, USA) which was averaging the measures over 5000 acquisitions. The signals were post-processed using the Matlab software (The MathWorks, Natick, MA, USA) by computing the Hilbert transform modulus of the signal and converting it into a line of color.

The ultrasound signal was simultaneously recorded from the echoes on the transducer.

\section{RESULTS}

The figure (6) shows respectively the ultrasound image and the electrical impedance image of the gelatin 


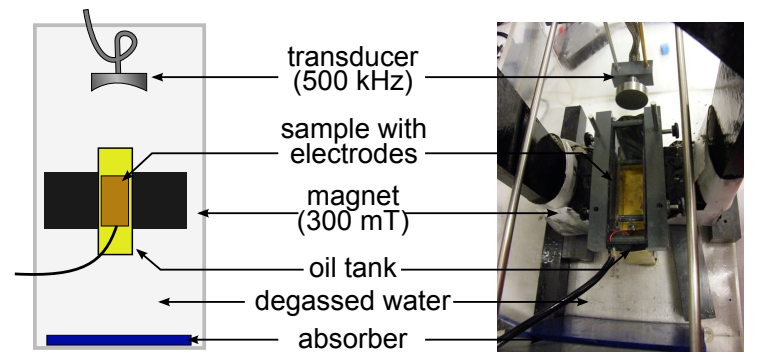

FiguRe 3 - A transducer is emitting ultrasound in a sample placed in an oil tank in the middle of a magnetic field. The induced electrical current is received by two electrodes.

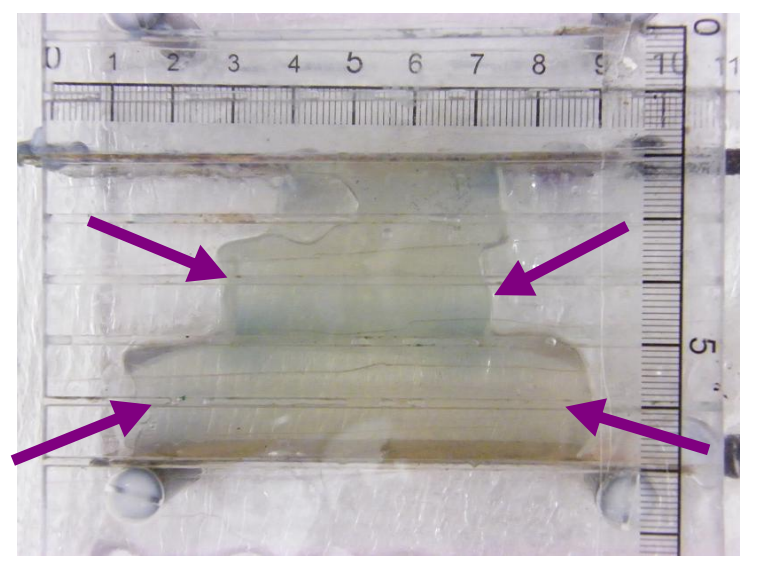

Figure 4 - Photo of salty gelatin sample. The sample is made of 2 blocks, one of $8 \times 2 \times 2 \mathrm{~cm}^{3}$ under the second of $4 \times 2 \times 3 \mathrm{~cm}^{3}$. Two electrodes are in contact above and under the sample. Arrows are indicating front and back interfaces.

phantom. The front and back interfaces are equally visible on both images. The relative amplitude of interface signal is however different, mostly due to magnetic field inhomogeneity.

The figure (7) shows respectively the ultrasound image and the electrical impedance image of the beef sample. The front interface can be seen on both images, although the lower one is less visible.

\section{DISCUSSION AND CONCLUSION}

A method to observe electrical conductivity gradients by combining ultrasound and magnetic field is tested experimentally.

The tested transducer is a standard single element ultrasonic therapy transducer. The magnetic field is created by a permanent magnet with sufficient air gap to insert a tissue sample. The signal has been shown to be little influenced by the electrodes positions [15. The oil bath prevents any electrical contact between the sample and the transducer; in practical applications it could easily be placed around the

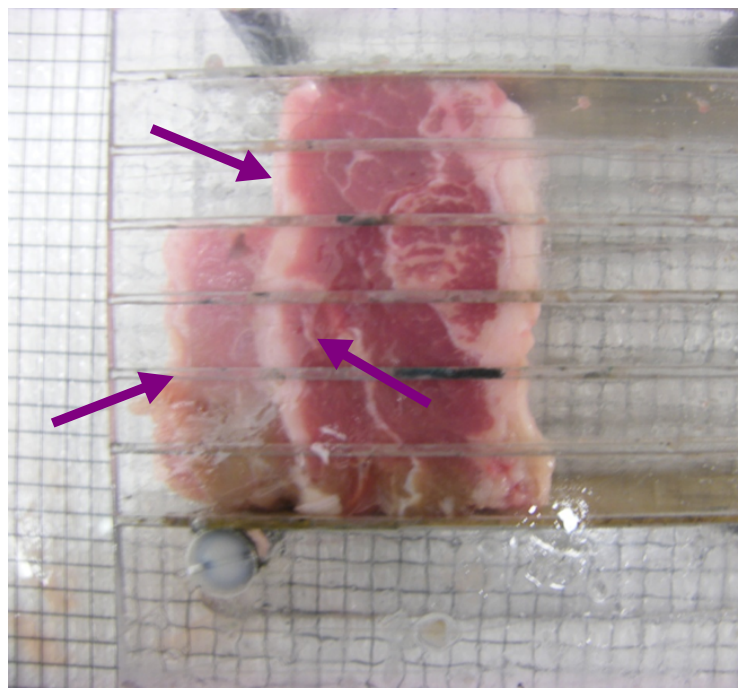

Figure 5 - Photo of beef sample. The sample has an L-shape, $6 \mathrm{~cm}$ wide, $2 \mathrm{~cm}$ large and $6 \mathrm{~cm}$ high. Two electrodes are in contact above and under the sample. Squares are $5 \mathrm{~mm}$ wide. Arrows are indicating two front interfaces and the fat layer.

transducer rather than around the patient. The experiment showed images of quality comparable to the one of the ultrasound images taken in the same conditions. The combination of ultrasonography and Lorentz Force Electrical Impedance Tomography with ultrasonically-induced Lorentz force can be made easily with the same material. Elements like fat layers which are hardly visible here in the ultrasonic image can be observed in the Lorentz Force Electrical Impedance Tomography image. The technique has potentially the spatial resolution of the ultrasound wavelength 12, allowing the observation of small inhomogeneities and can help thus revealing pathologies like cancer by detecting tumorous tissues where other techniques fail to do so.

Image quality could be improved with a higher ultrasound frequency and a thinner beam. The compatibility of ultrasound imaging with MRI [10] shows that it is possible to use much stronger magnetic fields, which would increase the intensity of the induced electrical current, and thus the signal to noise ratio. Moreover, even if the magnetic field homogeneity is not as critical in this technique as in MRI [16, a more homogeneous magnetic field would provide sharper images of the interfaces. The technique can also be used in a reverse mode [21, with an electrical current applied in a tissue submitted to a magnetic field, which leads to ultrasound wave 22 and was recently applied to human tissue ex-vivo [11. It is nevertheless hard to say which of these techniques would be the most useful for biomedical imaging [19]. 

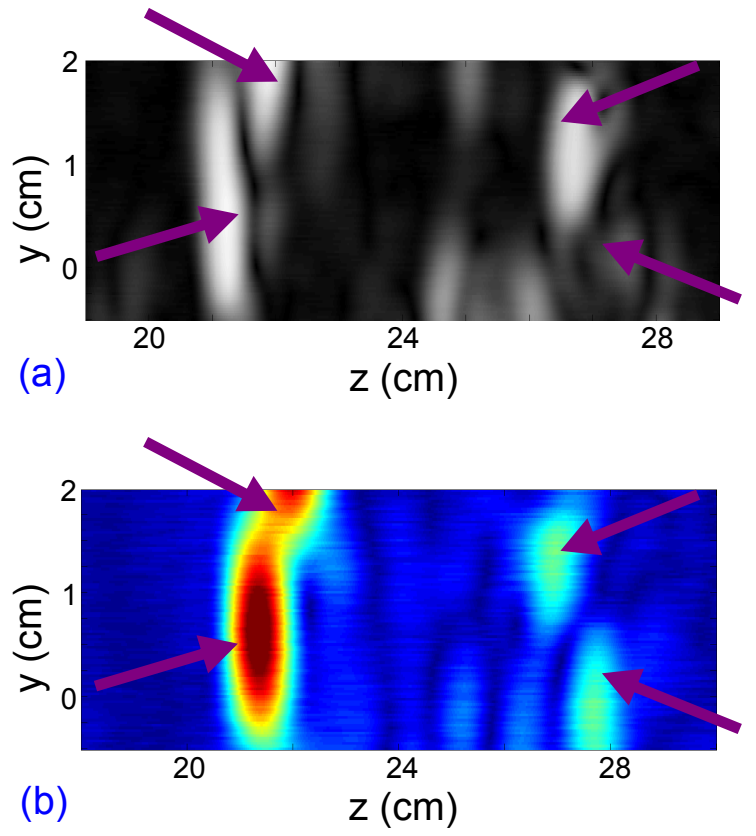

FiguRE 6 - (a) Ultrasound image of the gelatin sample. (b) Electrical impedance image of the gelatin sample. Arrows are indicating the interfaces shown on the photograph.

\section{ACKNOWLEDGMENTS}

The authors would like to thank Amalric Montalibet for its pioneer work on the subject, Rémi Souchon for his advices and Alexandre Petit for theory suggestions. No conflict of interest is declared.

\section{RÉFÉRENCES}

[1] Habib Ammari, Yves Capdeboscq, Hyeonbae Kang, Anastasia Kozhemyak, et al. Mathematical models and reconstruction methods in magnetoacoustic imaging. Euro. J. Appl. Math, 20 :303317,2009

[2] Habib Ammari and Hyeonbae Kang. Reconstruction of small inhomogeneities from boundary measurements. Springer, 2004.

[3] Margaret Cheney, David Isaacson, and Jonathan C Newell. Electrical impedance tomography. SIAM review, 41(1) :85-101, 1999.

[4] Leszek Filipczynski. Absolute measurements of particle velocity, displacement or intensity of ultrasonic pulses in liquids and solids. Acustica, $21: 173-180,1969$.

[5] Camelia Gabriel, Sami Gabriel, and E Corthout. The dielectric properties of biological tissues : I. literature survey. Physics in medicine and biology, 41(11) :2231, 1996.

[6] Pol Grasland-Mongrain, Jean-Martial Mari, Bruno Gilles, Jean-Yves Chapelon, and Cyril La-
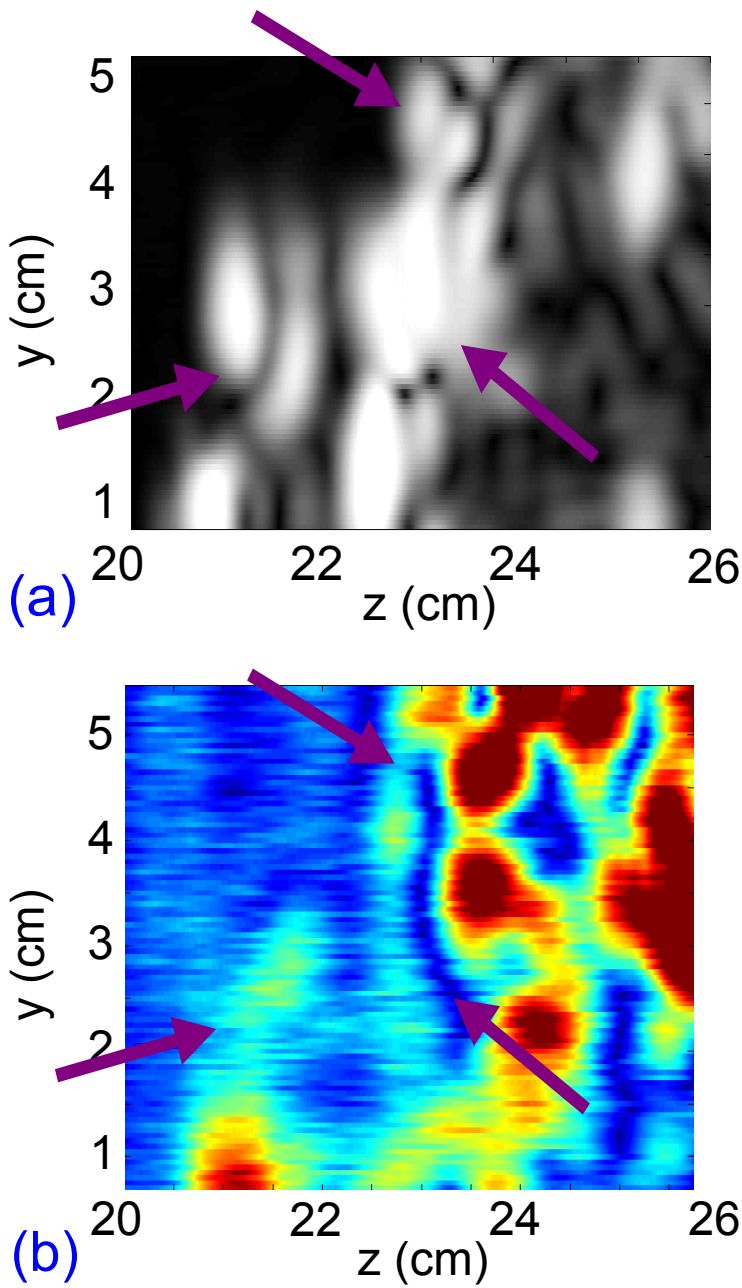

FIGURE 7 - (a) Ultrasound image of the beef sample. (b) Electrical impedance image of the beef sample. Arrows are indicating the interfaces shown in the photograph.

fon. Electromagnetic hydrophone with tomographic system for absolute velocity field mapping. Applied Physics Letters, 100(24) :243502-243502, 2012.

[7] Pol Grasland-Mongrain, Jean-Martial Mari, Bruno Gilles, and Cyril Lafon. Electromagnetic hydrophone for high-intensity focused ultrasound (hifu) measurement. Proceedings of Meetings on Acoustics, 2013 (in press).

[8] Dieter Haemmerich, ST Staelin, JZ Tsai, S Tungjitkusolmun, DM Mahvi, and JG Webster. In vivo electrical conductivity of hepatic tumours. Physiological measurement, 24(2) :251, 2003.

[9] S Haider, A Hrbek, and Y Xu. Magneto-acoustoelectrical tomography : a potential method for imaging current density and electrical impedance. Physiological measurement, 29(6) :S41, 2008. 
[10] Pascal Haigron, Jean-Louis Dillenseger, Limin Luo, and Jean-Louis Coatrieux. Image-guided therapy : evolution and breakthrough [a look at]. Engineering in Medicine and Biology Magazine, IEEE, 29(1) :100-104, 2010.

[11] Gang Hu, Erik Cressman, and Bin He. Magnetoacoustic imaging of human liver tumor with magnetic induction. Applied physics letters, 98 :023703, 2011.

[12] MR Islam and BC Towe. Computer image reconstruction of bioelectric currents from magnetoacoustic measurements. In Engineering in $\mathrm{Me}$ dicine and Biology Society, 1988. Proceedings of the Annual International Conference of the IEEE, pages 441-442. IEEE, 1988.

[13] Jean Martial Mari, Thierry Blu, Olivier Bou Matar, Michael Unser, and Christian Cachard. A bulk modulus dependent linear model for acoustical imaging. The Journal of the Acoustical Society of America, 125 :2413, 2009.

[14] Jean Martial Mari, Kathryn Hibbs, Eleanor Stride, Robert J Eckersley, and Meng Xing Tang. An approximate nonlinear model for time gain compensation of amplitude modulated images of ultrasound contrast agent perfusion. Ultrasonics, Ferroelectrics and Frequency Control, IEEE Transactions on, 57(4) :818-829, 2010.

[15] Amalric Montalibet. Etude du couplage acoustomagnétique : détection des gradients de conductivité électrique en vue de la caractérisation tissulaire. PhD thesis, Institut Nationale des Sciences Appliquées de Lyon, 2002.

[16] Amalric Montalibet, Jacques Jossinet, and Adrien Matias. Scanning electric conductivity gradients with ultrasonically-induced lorentz force. Ultrasonic imaging, 23(2) :117-132, 2001.

[17] Amalric Montalibet, Jossinet Jossinet, Adrien Matias, and Dominique Cathignol. Electric current generated by ultrasonically induced lorentz force in biological media. Medical and Biological Engineering and Computing, 39(1) :15-20, 2001.

[18] B.J. Roth, Jr. Wikswo, J.P., Han Wen, and R.S. Balaban. Comments on "hall effect imaging" [with reply]. Biomedical Engineering, IEEE Transactions on, 45(10) :1294-1296, 1998.

[19] Bradley J Roth. The role of magnetic forces in biology and medicine. Experimental Biology and Medicine, 236(2) :132-137, 2011.

[20] Bradley J Roth, Peter J Basser, and John P Wikswo. A theoretical model for magnetoacoustic imaging of bioelectric currents. IEEE transactions on biomedical engineering, 41(8) :723-728, 1994.
[21] Han Wen, Jatin Shah, and Robert S Balaban. Hall effect imaging. Biomedical Engineering, IEEE Transactions on, 45(1) :119-124, 1998.

[22] Yuan Xu and Bin He. Magnetoacoustic tomography with magnetic induction (mat-mi). Physics in medicine and biology, 50(21) :5175, 2005. 